\title{
The glycosyltransferase ST3GAL2 is regulated by miR-615-3p in the intestinal tract of Campylobacter jejuni infected mice
}

\author{
De Xi' ${ }^{1}$, Lukas Hofmann ${ }^{1}$, Thomas Alter ${ }^{2}$, Ralf Einspanier ${ }^{1}$, Stefan Bereswill ${ }^{3}$, Markus M. Heimesaat ${ }^{3}$, \\ Greta Gölz ${ }^{2+}$ and Soroush Sharbati ${ }^{1 *+}$ (1)
}

\begin{abstract}
Background: Campylobacter jejuni (C. jejuni) infections are of increasing importance worldwide. As a typical mucosal pathogen, the interaction of $C$. jejuni with mucins is a prominent step in the colonisation of mucosal surfaces. Despite recent advances in understanding the interaction between bacterial pathogens and host mucins, the mechanisms of mucin glycosylation during intestinal C. jejuni infection remain largely unclear. This prompted us to identify relevant regulatory networks that are concerted by miRNAs and could play a role in the mucin modification and interaction.

Results: We firstly used a human intestinal in vitro model, in which we observed altered transcription of MUC2 and TFF3 upon C. jejuni NCTC 11168 infection. Using a combined approach consisting of in silico analysis together with in vitro expression analysis, we identified the conserved miRNAs miR-125a-5p and miR-615-3p associated with MUC2 and TFF3. Further pathway analyses showed that both miRNAs appear to regulate glycosyltransferases, which are related to the KEGG pathway 'Mucin type O-glycan biosynthesis.' To validate the proposed interactions, we applied an in vivo approach utilising a well-established secondary abiotic IL-10 ${ }^{-/-}$mouse model for infection with C. jejuni 81-176. In colonic tissue samples, we confirmed infection-dependent aberrant transcription of MUC2 and TFF3. Moreover, two predicted glycosyltransferases, the sialyltransferases ST3GAL1 and ST3GAL2, exhibited inversely correlated transcriptional levels compared to the expression of the identified miRNAs miR-125a-5p and miR-615-3p, respectively. In this study, we mainly focused on the interaction between miR-615-3p and ST3GAL2 and were able to demonstrate their molecular interaction using luciferase reporter assays and RNAi. Detection of ST3GAL2 in murine colonic tissue by immunofluorescence demonstrated reduced intensity after C. jejuni 81-176 infection and was thus consistent with the observations made above.
\end{abstract}

Conclusions: We report here for the first time the regulation of glycosyltransferases by miRNAs during murine infection with C. jejuni 81-176. Our data suggest that mucin type O-glycan biosynthesis is concerted by the interplay of miRNAs and glycosyltransferases, which could determine the shape of intestinal glycosylated proteins during infection.

Keywords: Campylobacter jejuni, Mucin, miRNA, Glycosyltransferase, Host cell response

\footnotetext{
*Correspondence: soroush.sharbati@fu-berlin.de

†Soroush Sharbati and Greta Gölz contributed equally to this work

1 Institute of Veterinary Biochemistry, Freie Universität Berlin, Berlin, Germany

Full list of author information is available at the end of the article
}

\section{Background}

The colonic mucosal layer is a dynamic and constantly renewing physical barrier providing protective functions through viscoelastic properties of mucins [1]. MUC2 is characterised by containing repeating domains rich in the amino acids proline, threonine and serine (PTS

(C) The Author(s) 2021. This article is licensed under a Creative Commons Attribution 4.0 International License, which permits use, sharing, adaptation, distribution and reproduction in any medium or format, as long as you give appropriate credit to the original author(s) and the source, provide a link to the Creative Commons licence, and indicate if changes were made. The images or other third party material in this article are included in the article's Creative Commons licence, unless indicated otherwise in a credit line to the material. If material is not included in the article's Creative Commons licence and your intended use is not permitted by statutory regulation or exceeds the permitted use, you will need to obtain permission directly from the copyright holder. To view a copy of this licence, visit http://creativeco mmons.org/licenses/by/4.0/. The Creative Commons Public Domain Dedication waiver (http://creativecommons.org/publicdomain/ zero/1.0/) applies to the data made available in this article, unless otherwise stated in a credit line to the data. 
domains), which are densely $O$-glycosylated by the glycosylation machinery of the Golgi apparatus [2]. The PTS domain decorated with abundant $O$-glycans (mucin type $O$-glycans) forms a special "mucin domain" that makes up approximately $80 \%$ of the MUC2 mass and gives mucins extended conformation and furthermore, contributes to the structural integrity and complexity of mucins [2]. Assembled MUC2 polymers are stored in a condensed way in granules within goblet cells before being released into intestinal lumen. Once released, these large polymers are expanded in volume to form the mucus gel and provide the structural basis of the mucus layer [2]. Besides the mucins, also mucin-associated proteins such as the trefoil factor family (TFF) peptides are important for the restitution and integrity of the intestinal mucosal layer [3]. TFF3 is co-secreted with MUC2 by colonic goblet cells and usually acts in a cooperative manner with MUC2 ensuring elasticity and viscosity of MUC2 [3, 4].

The mucus layer provides protection from enteropathogens in several ways. This essential physical barrier hinders pathogenic as well as commensal bacteria to access the underlying intestinal epithelium. Previous studies have found that the lack of MUC2 and TFF3 resulted in pronounced defects in mouse intestinal homeostasis and enhanced susceptibility towards colitis, emphasising the importance of the mucus layer $[1,5,6]$. Moreover, the role of glycosylation of MUC2 in protecting the host from intruding bacteria has gained some appreciation in recent years. Application of knockout-based experiments led to reports of several glycosyltransferases involved in the biosynthesis and structural formation of core $O$-glycans of MUC2. For example, mice deficient in $\mathrm{N}$-acetylglucosaminyltransferase (GCNT2) displayed an overall altered mucin composition and exhibited a defective mucus barrier function, given that GCNT2 initiates core 2 derived $O$-glycan branching which is the basis of mucin core structure 2 and 4 [7]. This indicates that $O$-glycosylation of MUC2 has important implications in forming the skeleton of the mucus layer and maintaining integrity of mucus barrier. Nevertheless, the mechanisms that regulate post-transcriptional modification of $O$-linked glycans on mucins like sialylation or fucosylation remain still unclear.

$O$-glycans are oligosaccharide moieties abundant on the cell surface proteins of both host and pathogens forming a critical interface with the biological milieu and profoundly influence the pathogen-host interaction $[8,9]$. $O$-glycans on mucin proteins are used as ligands for bacterial adhesins and can prevent bacteria to access epithelial cells by binding and hampering them in mucus [10]. On the other hand, specific binding via "glycan-glycan talk" constitutes an important mechanism for bacteria to mediate adhesion and invasion of host cells [8].
During infection, bacterial pathogens can mediate host cell glycan modification utilising a broad range of glycosyltransferases and glycosidases, which in turn facilitates host adaption and adhesion and promotes access to glycans as carbon sources $[8,11]$. Campylobacter jejuni (C. jejuni) is a major cause of bacterial food-borne disease worldwide and capable to induce gastroenteritis and irritable bowel disease in humans [12]. In the course of colonisation, the binding of $C$. jejuni to the host mucins is mediated by glycan-glycan interaction $[8,11]$. Nevertheless, only little is known about the mechanisms of $C$. jejuni interaction with glycans of host mucosal surfaces and corresponding regulatory pathways involving mucinmodifying enzymes such as glycosyltransferases.

Recent efforts in non-coding RNA (ncRNA) research have expanded our understanding of mechanisms that regulate gene expression. MicroRNA (miRNA) belong to a well-studied class of ncRNAs, which can regulate gene expression through binding to complementary target sites of e.g. messenger RNAs (mRNAs), thereby initiating their degradation or suppression of translation [13]. Based on the fact that a single miRNA can target different genes and a single target gene can be coordinately regulated by different miRNAs, complex regulatory networks are formed to concert diverse biological processes including cell differentiation, proliferation, apoptosis or immune response [13, 14]. Our work as well as that of other research groups showed that miRNAs play a significant role in bacterial infections [14-17]. For example, the miR-125 family was shown to play an important role in immune response to bacterial as well as viral infections. Increased expression of miR-125b induced by lipomannan from virulent Mycobacterium tuberculosis can destabilise the transcript of tumour necrosis factor and therefore block its biosynthesis [17]. Moreover, work form Zhou et al. indicated that during Helicobacter pylori (H. pylori) infection, MUC2 expression could be post-transcriptionally affected by the cooperation of the lncRNA AF147447 with miR-34c [18]. Interestingly, Singh et al. found that under the influence of the endogenous microbiota, murine caecal miRNA signatures vary. Thus, intestinal barrier function may be affected by targeting genes that encode junctional proteins or glycosylation enzymes [19]. Furthermore, growing evidence has shown that miRNAs are major regulators of the glycome, controlling the level of glycan biosynthesis enzymes and playing a pivotal role in modulating and controlling glycosylation [20]. Kurcon et al. have utilised the glycogene target network of miR-200-family to identify three glycosylation enzymes (ST3 $\beta$-Galactoside $\alpha$-2,3-Sialyltransferase 5 (ST3GAL5), ST6 $N$-acetyl-galactosaminide $\alpha$-2,6-Sialyltransferase 5 (ST6GALNAC5) and $\beta 1,3$-Glucosyltransferase (B3GLCT), controlling 
epithelial-to-mesenchymal transition in MDA-MB-231 cells [21]. Nevertheless, the number of validated glycogene-miRNA-interactions is still limited [20].

In this work, we applied in vitro and in vivo approaches to address which intestinal miRNA may be associated with MUC2 modification during C. jejuni infection and what potential regulatory networks exist in this context. Firstly, we confirmed that $C$. jejuni NCTC 11168 infection induced an altered transcription of MUC2 and the cosecreted peptide TFF3 in human intestinal epithelial cells in vitro. After in silico prediction of mucin-associated regulatory networks, we secondly examined the expression of two miRNAs and two glycosyltransferase genes in murine colonic tissue and found C. jejuni 81-176-mediated dysregulation of their transcription. Thirdly, we focused on the ST3 $\beta$-Galactoside $\alpha$-2,3-Sialyltransferase 2 (ST3GAL2) and miR-615-3p interaction given their distinguished anti-correlated transcription upon $C$. jejuni 81-176 infection in vivo and were able to show that miR615-3p interacts with ST3GAL2 targeting its cellular concentrations.

\section{Results}

C. jejuni infection alters the transcription of MUC2 and TFF3 in human intestinal epithelial cells in vitro

MUC2 and TFF3 expression undergo changes during intestinal diseases caused by pathogenic bacteria, for instance [22]. In order to address this and find associated regulatory networks in response to $C$. jejuni infection, we determined the transcription of MUC2 and TFF3 in the human intestinal epithelial cell line HT-29/B6 following C. jejuni NCTC 11168 infection. The relative fold differences of MUC2 and TFF3 mRNA levels were calculated and revealed an approximately 2-fold up-regulation $(P \leq 0.01)$ of both factors relatively early (i.e., $1 \mathrm{~h})$ post infection (p.i.) (Fig. 1A and B). Thereafter, a consistent decrease of MUC2 transcription was observed in the course of infection until $24 \mathrm{~h}$ p.i., whereas no significant differences could be assessed when comparing the infected and non-infected control groups at 5 and $24 \mathrm{~h}$ p.i. (Fig. 1A). Upon infection, the transcript level of TFF3 showed an early peak followed by a 0.61 -fold decline at $5 \mathrm{~h}$ p.i. and a return to control levels until $24 \mathrm{~h}$ p.i. (Fig. 1B). Hence, both MUC2 and TFF3 mRNA expression significantly increased immediately after $C$. jejuni infection and subsequently decreased to levels that were comparable to non-infected controls.

\section{Prediction of MUC2-TFF3-associated miRNAs}

Recent data $[15,16]$ showed that regulatory miRNA networks influence the host response to bacterial infections. Therefore, we speculated that besides the alterations in the transcription of MUC2 and TFF3 also post-translational modification of MUC2 might be changed which might be due to pathogen-mediated manipulation of regulatory networks composed of mRNA and miRNAs. To address this, we used miRmap [23] with a score of above 50-as described previously [24] - to identify miRNAs supposed to target MUC2 and TFF3, respectively. After intersecting both lists of miRNAs, we identified 12 miRNAs (Fig. $1 \mathrm{C}$ and Additional file 1) that could mutually target MUC2 and TFF3. From this, we selected eight miRNAs, which were conserved among human, mouse and rat. To assess their involvement in $C$. jejuni infection in vitro, we evaluated the expression of miR-125a-5p, miR-615-3p, miR-671-5p and miR-320 family in C. jejuni NCTC 11168 infected HT-29/ B6 cells. The RT-qPCR results indicated that only miR125a-5p and miR-615-3p showed regulated expression within $24 \mathrm{~h}$ p.i. Immediately after infection, miR-125a-5p was significantly downregulated (0.72-fold, $P \leq 0.05)$, whereas its expression returned to baseline values afterwards (Fig. 1D). MiR-615-3p expression showed a similar regulation with a 0.84 -fold decrease $(P \leq 0.05)$ and its transcript level was rather consistent until $24 \mathrm{~h}$ p.i. (0.83fold, $P \leq 0.05)($ Fig. $1 \mathrm{E}$ ). Both miRNAs possessed anticorrelated expression with MUC2 and TFF3 mRNAs. As shown in Fig. 1F, miR-320 expression was barely affected along the entire course of infection. Of note, the miR671-5p level was below detection limit (data not shown).

\section{Identification of regulatory networks for in vivo analysis}

As shown above, an inverse correlation between two predicted miRNAs and MUC2 as well as TFF3 was observed in the early phase of C. jejuni NCTC 11168 infection in human cells. This indicated a regulatory importance of miR-125a-5p and miR-615-3p on related pathways upon C. jejuni infection. Therefore, we expanded our analysis to determine genes that are relevant to the post-transcriptional modification of MUC2 and might be regulated by the same miRNAs in the context of $C$. jejuni infection in vivo applying secondary abiotic mice [25]. For this purpose, we predicted the murine targets of both miR-125a-5p and miR-615-3p by means of another round of miRmap analysis (Additional file 1). After intersecting both lists of targets, we determined a gene list that was supposed to be regulated by both miRNAs. Using this list, we performed a KEGG pathway enrichment [26] by means of Cytoscape with the app ClueGO [27] (Fig. 2A, Additional file 2). In total, target genes were significantly assigned to seven KEGG terms and we found that four target genes were significantly $(P \leq 0.05)$ enriched in the KEGG pathway "mmu00512: namely, Mucin type $O$-glycan biosynthesis", which corresponded to $14.3 \%$ of the associated genes in this term. They consisted of ST3 $\beta$-Galactoside $\alpha$-2,3-Sialyltransferase 1 (ST3GAL1), ST3 


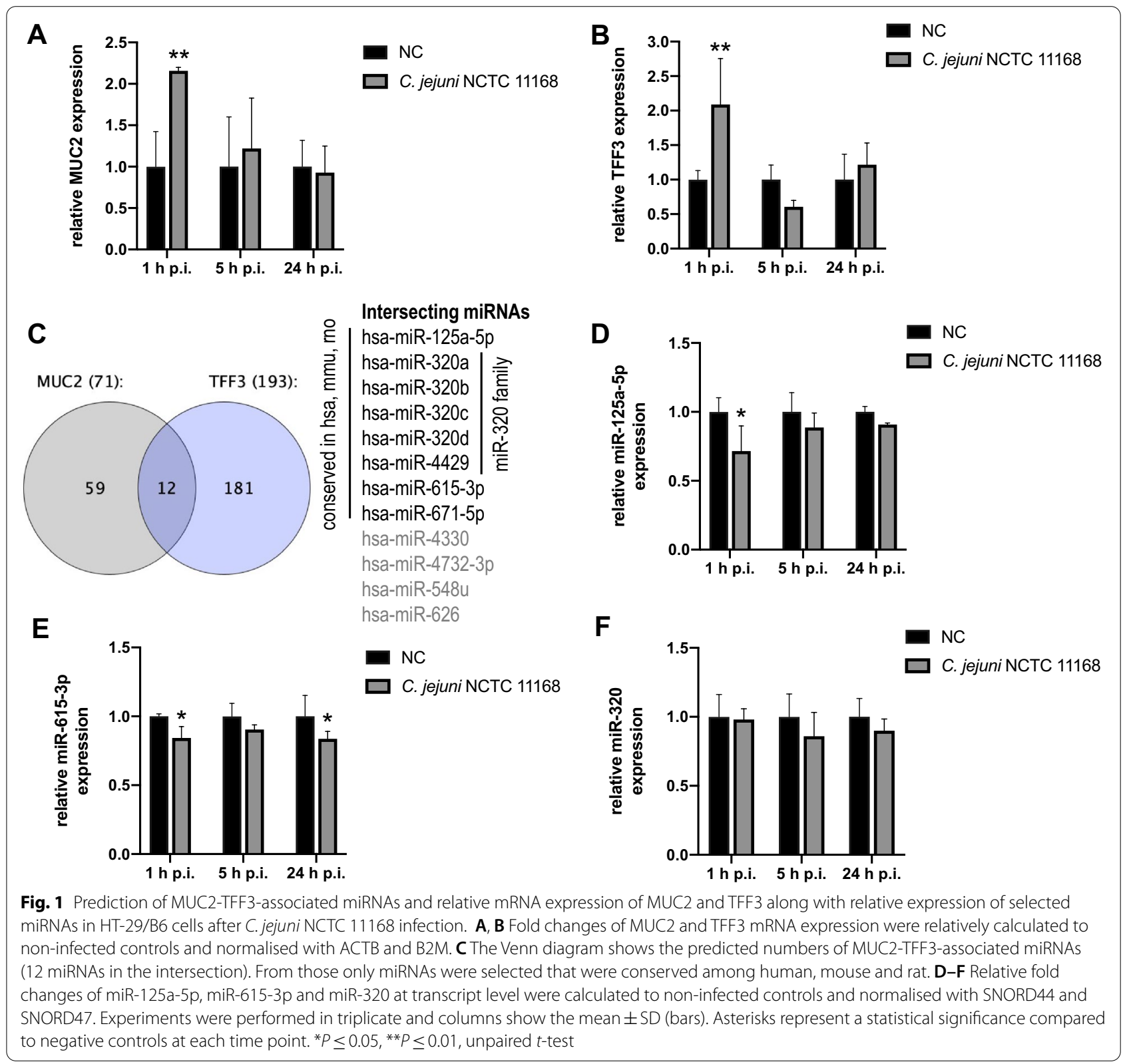

$\beta$-Galactoside $\alpha$-2,3-Sialyltransferase 2 (ST3GAL2), $\beta 1,6$ $N$-acetylglucosaminyltransferase 1 (GCNT1), and $\beta 1,6$ $\mathrm{N}$-acetylglucosaminyltransferase 4 (GCNT4). These four glycosyltransferases were identified as potentially regulated candidates of both miRNAs upon $C$. jejuni infection in vivo (Fig. 2A).

\section{Expression analysis of interacting miRNAs and mRNAs} in the colon of $C$. jejuni infected secondary abiotic IL-10 $10^{-1-}$ mice

In addition to the early stage of infection with $C$. jejuni NCTC 11168 investigated in human cells, we further surveyed the later stage of infection with $C$. jejuni strain 81-176 in a well-established murine model [25] to assess the biological relevance of the observed regulations and predicted interactions in vivo. Therefore, the relative transcription of MUC2 and TFF3 was measured in colonic tissue samples taken at day 6 following peroral infection of secondary abiotic IL- $10^{-/-}$mice. As shown in Fig. 2B, C. jejuni 81-176 infection resulted in a pronounced decrease of TFF3 transcription when compared to naïve controls $(0.38$ fold change, $P \leq 0.0001$, whereas the MUC2 transcription was slightly decreased upon infection $(0.80$-fold 


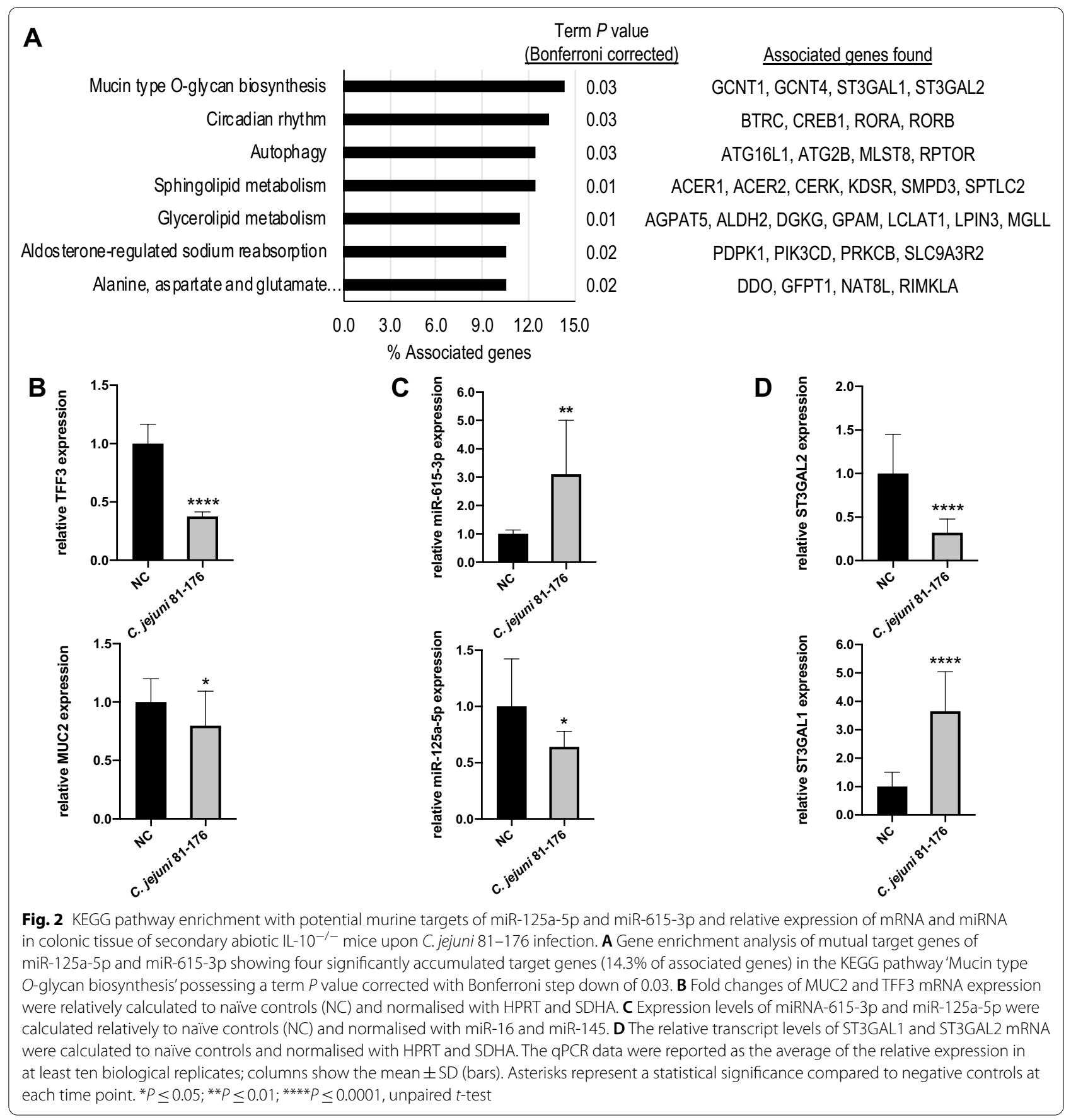

change, $P \leq 0.05)$. Secondly, we addressed whether the identified miRNAs were also regulated during $C$. jejuni 81-176 infection in mice, especially at the late stage of infection. As demonstrated in Fig. 2C, infected (secondary abiotic IL-10 $0^{-1-}$ ) mice exhibited more than triple miR-615-3p transcript levels $(P \leq 0.01)$ compared to non-infected control mice. In contrast, the miR125a-5p transcription decreased at the late stage of $C$. jejuni infection (0.64-fold changes, $P \leq 0.05$; Fig. $2 \mathrm{C}$ ). As pathway analysis predicted that glycosyltransferases involved in the mucin type $O$-glycan biosynthesis might be regulated by the identified miR-125a-5p and miR-615-3p, we were intrigued to unravel whether predicted targets ST3GAL1 and ST3GAL2 are dysregulated in an anti-correlated manner. Therefore, we examined their transcription in identical samples and 
observed a strongly reduced transcription (0.32-fold changes, $P \leq 0.0001)$ of ST3GAL2 elicited by $C$. jejuni 81-176 infection (Fig. 2D), which was anti-correlated with the miR-615-3p expression. In contrast, ST3GAL1 transcription was markedly enhanced (3.65-fold, $P \leq 0.0001$ ) when compared to naïve controls (Fig. 2D) and exhibited inverse correlation with miR-125a-5p.

\section{ST3GAL2 is down-regulated in the murine epithelial cell line CMT 93 after RNAi using miR-615-3p mimic}

MiRNAs are known to play a pivotal role in mRNA degradation or transcriptional repression by binding to their targets [13]. The results of the in vivo experiments suggested that the mRNA level of ST3GAL2 might be modulated by miR-615-3p and miR-125a-5p may play a regulatory role on ST3GAL1 transcription in colonic tissue after $C$. jejuni 81-176 infection. In this study, we only focused on the interaction of ST3GAL2 and miR-615-3p. To explore whether miR-615-3p was responsible for the decreased ST3GAL2 mRNA levels, we performed RNAi experiments using miR-615-3p mimics in the mouse rectum cell line CMT 93 considering nonsense transfected cells as controls. After RNAi, expression analysis of both miR-615-3p and its proposed target ST3GAL2 mRNA clearly revealed anti-correlated transcript levels, whereas the cellular miR-615-3p concentration was increased by 4.3 -fold $(P \leq 0.0001)$ (Fig. $3 \mathrm{~A})$, which was accompanied by a significant 0.74 -fold decrease in the ST3GAL2 mRNA level $(P \leq 0.05)$ (Fig. 3B).

Reporter assays confirm ST3GAL2 as a target of miR-615-3p To verify whether miR-615-3p directly binds to a target site within the $3^{\prime}$ UTR of ST3GAL2 and thereby repressively controls its post-transcriptional expression, we applied a dual luciferase reporter gene assay as described earlier [16]. Consequently, the $3^{\prime}$ UTR of murine ST3GAL2 was tested for binding sites of miR615-3p using RNAhybrid [28] as previously described [3]. As shown in Fig. 3C, two interaction sites were identified. CMT 93 cells were transfected with a luciferase reporter plasmid containing a combination of both target sites [24] (Additional file 3), miR-615-3p mimic and a normalisation plasmid (pTKCluc). As shown in Fig. 3D, miR-615-3p mimics repressed luciferase activity by around 0.65 -fold $(P \leq 0.05)$ compared to nonsense transfected controls. The 0.65 -fold decrease in luciferase activity corresponded to the decrease in ST3GAL2 transcript levels after RNAi using miR-615-3p mimics in CMT 93 (approximately 0.75-fold of nonsense control). Hence, our results revealed specific interactions between at least one of the ST3GAL2 binding sites and miR-615-3p.
ST3GAL2 protein levels in C. jejuni infected mice

After showing that miR-615-3p specifically binds to the ST3GAL2 target sites in vitro and that intestinal ST3GAL2 transcript levels decrease upon C. jejuni infection in vivo, we further analysed the protein levels of ST3GAL2 by means of immunofluorescence and Western blot analyses of colonic tissue samples derived from $C$. jejuni infected secondary abiotic IL-10 $10^{-/-}$mice. As representatively shown in Fig. $4 \mathrm{~A}$ and $\mathrm{B}$ and the Additional file 4, the immunofluorescent ST3GAL2 signal was much stronger in colon sections derived from non-infected as compared to C. jejuni-infected mice. However, the overall fluorescence signal in mouse colon sections stained with the anti-ST3GAL2 antibody was of low intensity. Furthermore, negative controls were analysed in serial sections on the same tissue area proving the specificity of detected signals (data shown in the Additional file 4). Moreover, C. jejuni infection of mice resulted in distinct histomorphological changes within the colon mucosa and lamina propria such as apoptosis of epithelial cells, villous blunting and irregular crypts as reported previously $[29,30]$.

In addition, we performed Western blot assays for ST3GAL2 using the same treated murine colonic tissue samples as for qPCR analysis. Compared to the significant decrease in ST3GAL2 expression at the mRNA level and the apparent decrease in immunofluorescence signal of the ST3GAL2 protein, we found only a slight trend towards down-regulation of ST3GAL2 at the protein level by Western blot in infected samples (not significant; Fig. 4C).

\section{Discussions}

The intestinal mucus layer can confine enteropathogens in the outer layer and exclude pathogens through intestinal peristalsis and mucus turnover to protect epithelial cells from attack by pathogens [31]. Despite the defensive system the mucus provides, enteric pathogens are able to breach this barrier by employing manifold strategies. For instance, $C$. jejuni is able to grow and thrive in the mucus layer and can negotiate this barrier by distinct motility [32]. However, the host responses triggered because of $C$. jejuni interaction with the mucosa are not yet fully understood at the molecular level. Accordingly, the mechanisms of whether and how the mucin glycoprotein pattern is modulated during intestinal $C$. jejuni infection and what this means for the interaction with the host are still largely unknown.

The gel-forming mucin MUC2 and the viscosity regulating trefoil factor TFF3 are essential for intestinal integrity. It has been reported that microbial infection can induce mucin secretion by goblet cells as a host response 
A

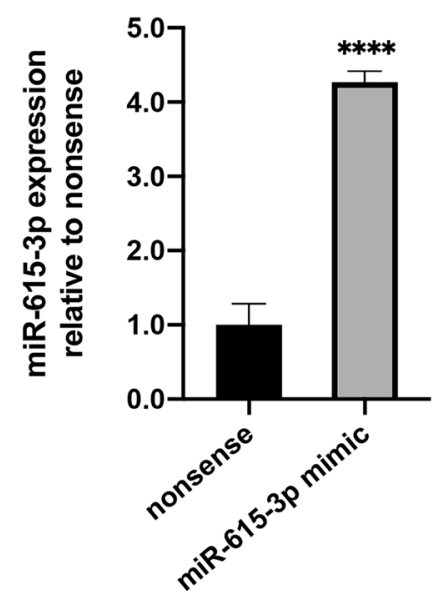

B

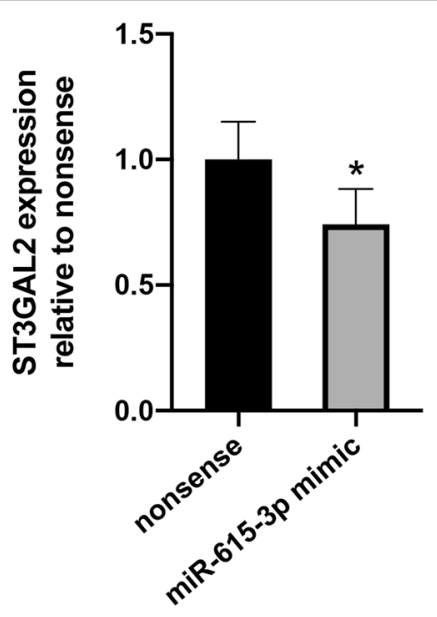

C

ST3GAL2 target site \#1

ST3GAL2

$5^{\prime}$

C GGGGCCU

$|:|: \mid 1$ :

CUCUGGG

$\operatorname{miR}-615-3 p$ 3' UUCUCC

mfe: $-29.3 \mathrm{kcal} / \mathrm{mol}$

\section{ST3GAL2 target site \#2}

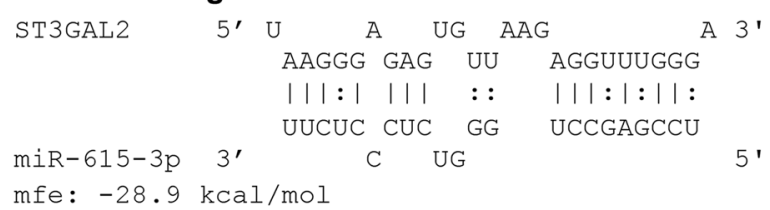

D

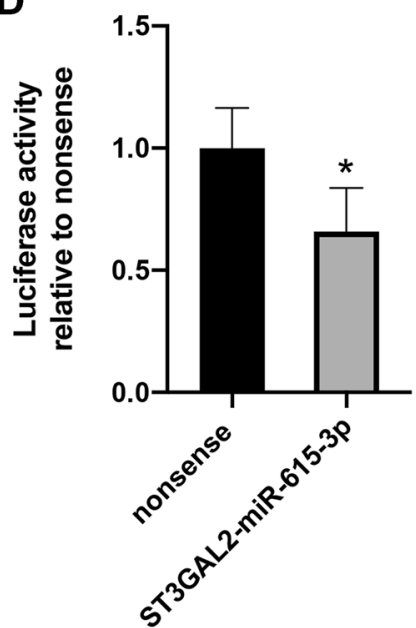

Fig. 3 Specific interaction of miR-615-3p with ST3GAL2 confirmed by RNAi and dual luciferase reporter assay. A, B RT-qPCR analysis for ST3GAL2 transcription in CMT 93 cells transfected with miR-615-3p mimics and nonsense miRNA as control. Fold change of ST3GAL2 and miR-615-3p was calculated relatively to nonsense control and normalised with HPRT, SDHA, B2M and miR-16, miR-145, respectively. Datasets are presented as means of three biological samples and double measurements \pm SD. C Identified target sites of ST3GAL2 and miR-615-3p were analysed with RNAhybrid. D Relative luciferase activity was determined to the nonsense miRNA mimic serving as control. Luciferase signal was assayed at $24 \mathrm{~h}$ post-transfection. Dataset was plotted as average values of at least four biological replicates with standard deviation from three measurements. Columns show the mean $\pm S D$ (bars). Asterisks represent a statistical significance compared to negative controls at each time point. ${ }^{*} P \leq 0.05$, ${ }^{* * * *} P \leq 0.0001$, unpaired $t$-test

to maintain and protect mucosal barrier [31]. Here, we observed significantly increased transcription of both MUC2 and TFF3 in cells derived from human colonic epithelial cell line HT-29/B6 immediately after C. jejuni NCTC 11168 infection. This is in agreement with a previous study, reporting increased transcriptional MUC2 expression upon Escherichia coli O157:H7 infection in human colon cells HT-29 [33]. In contrast to the upregulation of MUC2 and TFF3 mRNA in the initial stage of $C$. jejuni infection, we observed a slightly reduced transcription of MUC2 and profoundly decreased transcription of TFF3 in the colon of stably C. jejuni 81-176 infected mice (i.e., day 6 p.i.). Consistent with our observation, Bergstrom et al. found a slight reduction of MUC2 and TFF3 gene expression at 6 days p.i. and a significant decrease 10 days following murine Citrobacter rodentium infection [22]. According to our recent findings, $C$. jejuni strain NCTC 11168 and 81-176 exhibited comparable colonisation abilities and induced similar host responses with regard to histopathology and immune responses in secondary abiotic IL-10 ${ }^{-/-}$mice $[34,35]$. Therefore, we assume that the observed dysregulation of MUC2 and TFF3 upon in vitro and in vivo C. jejuni infection was 


\section{A Non-infected (negative control) IL-10-/- mice colon section}
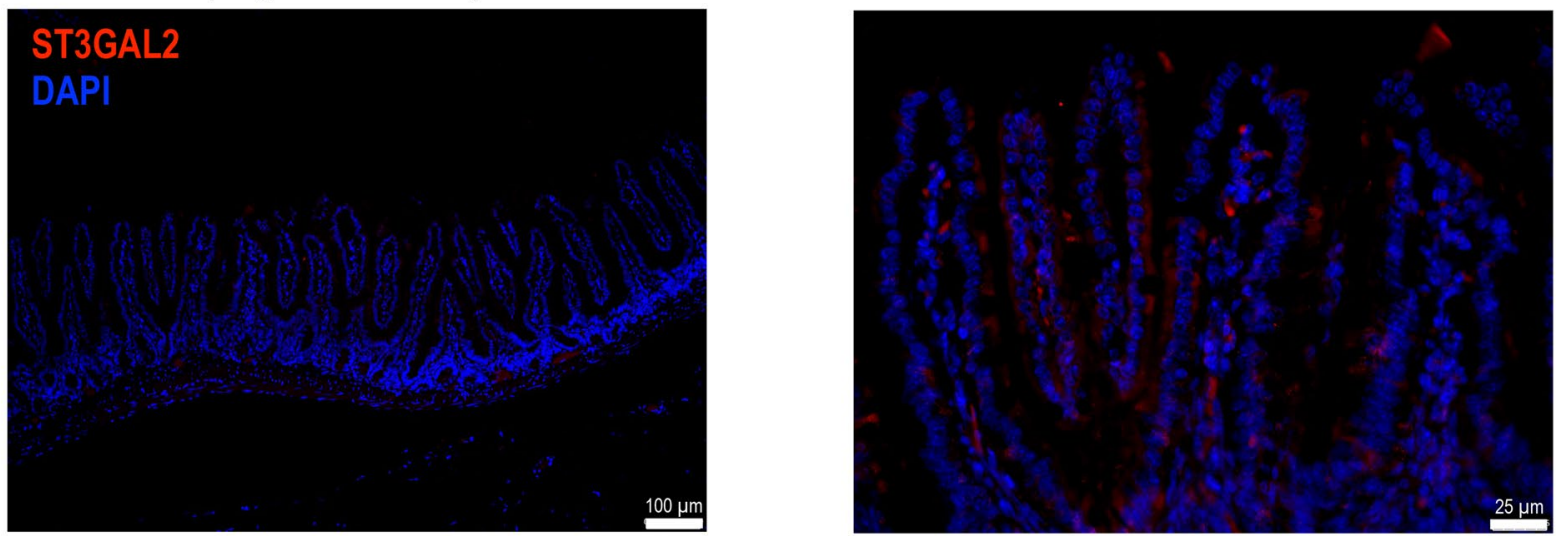

B

C. jejuni 81-176 infected IL-10/- mice colon section
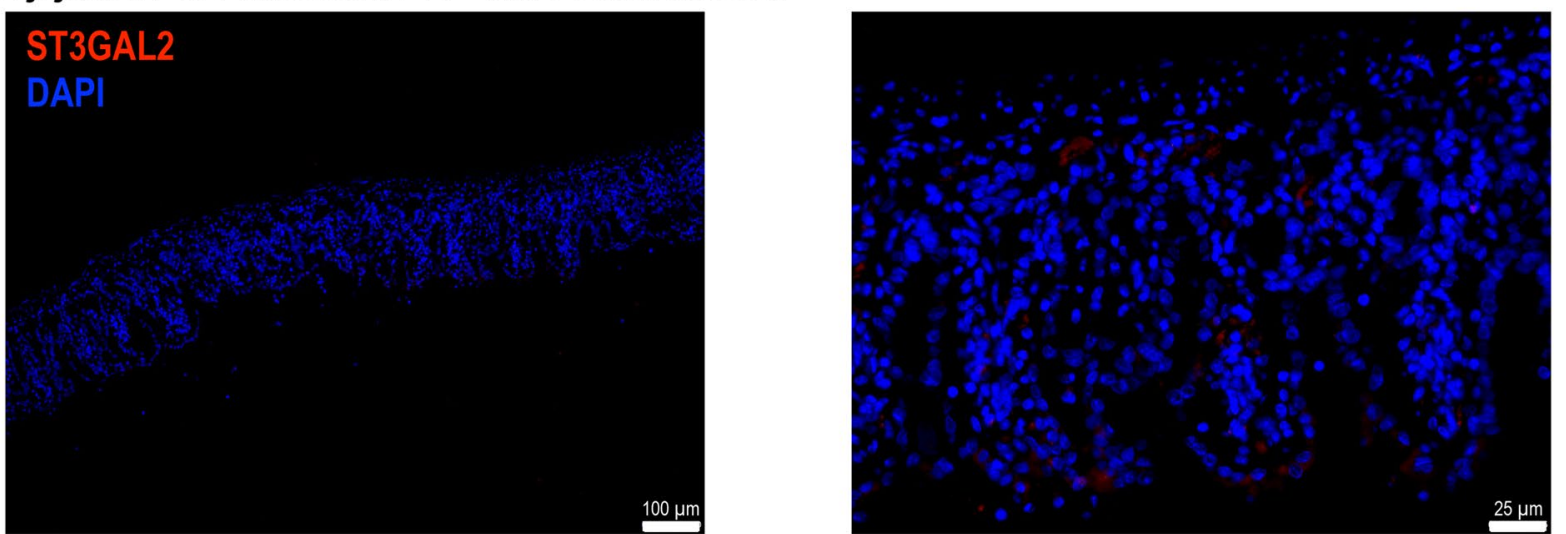

C
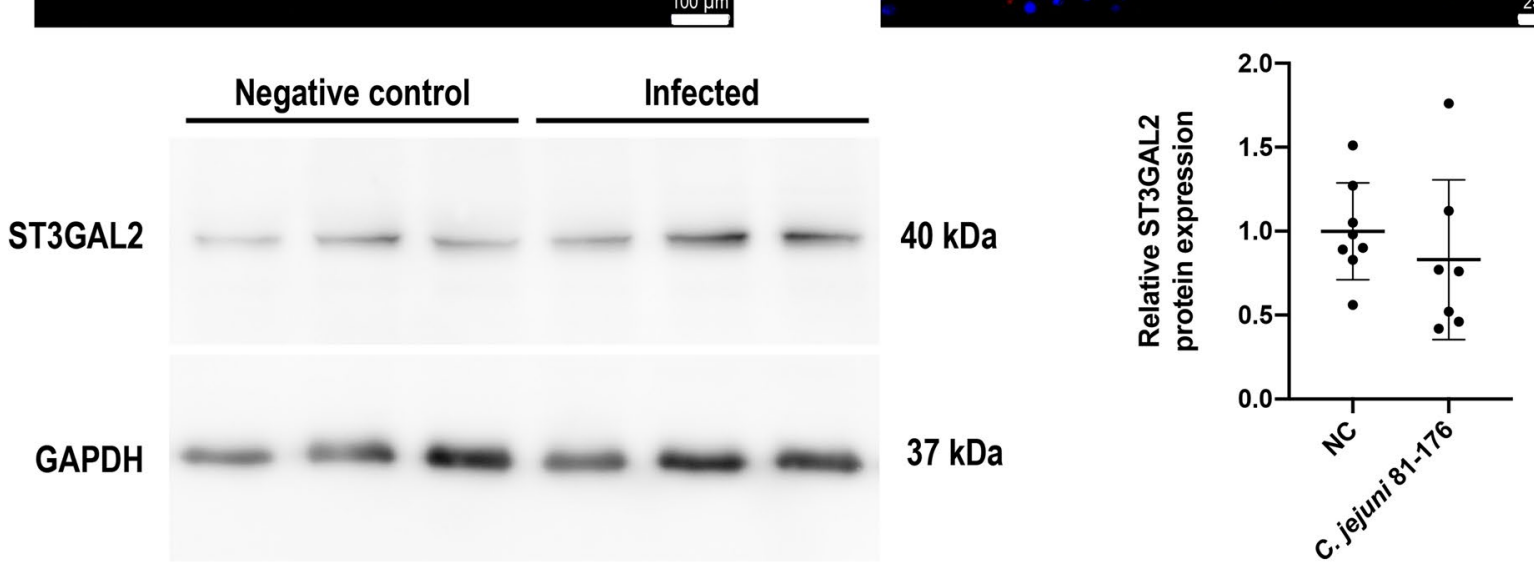

GAPDH

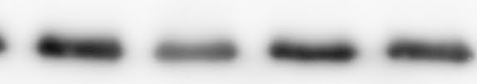

Fig. 4 Detection of protein expression of ST3GAL2 in colonic tissue of secondary abiotic IL-10-/- mice upon C. jejuni 81-176 infection. A,

B Representative micrographs comparing ST3GAL2 immunofluorescence results in the colonic tissue of naïve mice (A) and C. jejuni 81-176-infected mice (B). ST3GAL2 was immunofluorescently labelled in red and nuclei was stained with DAPI in blue. Micrographs at left side are at magnification $10 \times$ (Scale bar $100 \mu \mathrm{m}$ ) and right side at higher magnification 40× (scale bar $25 \mu \mathrm{m}$ ) and exposure time were the same in all sections. Data are representative for three biological replications. C Right panel exemplifies detection of ST3GAL2 from three individual replicates of infected and non-infected mouse colonic tissue via Western blotting. GAPDH is shown as respective loading reference. ST3GAL2 protein bands were quantified by densitometry relative to the respective GAPDH signals. Charts represent means of at least seven biological replicates \pm SD, unpaired $t$-test 
associated to the stage of infection rather than to the strains.

Evidence is emerging that miRNA-mediated regulation of mucins has important implications in inflammation and cancer biology [36]. For instance, miR-9 is responsible for gastric malignancies by targeting CDX2, conferring among others to enhanced proliferation of gastric cancer cells and MUC2 expression [36]. Further, miR-205 overexpression in CaCo- 2 cells resulted in the accumulation of mucus-secreting goblet cell-like cells and enhanced mucin production and MUC2 expression [37]. Based on our analysis, anti-correlated expression of miR-125a-5p and miR-615-3p was associated with the dysregulation of MUC2 and TFF3 transcription at the early stage of C. jejuni NCTC 11168 infection in vitro. Likewise, we observed anti-correlated transcription of miR-615-3p with MUC2 and TFF3 mRNA upon established $C$. jejuni 81-176 infection in vivo. These findings suggest that the alterations of mucin expression induced by $C$. jejuni are connected with miR-125a-5p and miR615-3p regardless of the strain. However, the direction of altered expressions seemed to be different at early and later stages of infection.

In this study, we aimed to build on the known role of MUC2 and TFF3 in intestinal bacterial infections and to identify novel regulatory aspects. For this purpose, we chose an approach that considers miRNAs as master regulators of those genes that belong to a metabolic or signalling pathway and interact accordingly. Here, we focused on the regulation of factors that are in the context of mucin modification and might be regulated by predicted miRNAs. Therefore, we concentrated our interest on target genes of miR-125a-5p and miR-615-3p, which may be related to metabolic changes of MUC2.

Upon enteric infection, quantity of host mucin may change by hypersecretion [31]. There can also be qualitative changes of mucins during infection including alterations in glycosylation. In this context, $H$. pylori was shown to change inflammation-associated mucin sialylation [31]. Previous studies have also observed altered degree of sulphation, sialylation and varied rate of glycosylation during intestinal inflammation as well as altered length and complexity of mucin glycans as a secondary effect to inflammation [38]. These alterations can further affect the adhesion of bacteria and the degradation of mucus by pathogens [38]. Thus, the interaction of mucus and pathogens seems to be a dynamic and complex process modulated by multiple factors [39]. Growing evidence has shown that miRNAs are major regulators of the glycome, playing a substantial role in modulating and controlling glycosylation [20]. However, our knowledge concerning the regulation of mucin modifying enzymes by miRNAs in bacterial infections is rather limited to date. According to our pathway analysis, several glycosyltransferases were potentially regulated by miR-125a-5p and miR-615-3p, from which we found two potential target genes (ST3GAL1, ST3GAL2) that are involved in mucin type $O$-glycan biosynthesis. $O$-glycans are the major glycans of mucins, which are generated via $O$-glycosylation and elongated or modified in a stepwise manner incorporating specific enzymes for sialylation, sulphation or acetylation [9]. ST3GAL1 and ST3GAL2 belong to a sialyltransferase family termed as ST3 $\beta$-Galactoside $\alpha-2,3-$ Sialyltransferase [40, 41]. ST3GAL1 predominantly adds sialic acid to the core $1 O$-glycan [40], while ST3GAL2 transfers sialic acid to the terminal galactose residues found in glycoconjugates and it is also known for its importance in the biosynthesis of gangliosides [41]. Our RT-qPCR results showed increased ST3GAL1 mRNA expression in murine colonic tissue 6 days after $C$. jejuni infection, while a profound reduction of ST3GAL2 mRNA level was observed. This suggests that $C$. jejuni infection affects the expression of sialyltransferase genes in the mouse colon. This might occur in a miRNA-dependent manner, since predicted miRNAs (miR-125a-5p and miR-615-3p) possessed anti-correlated expression. However, whether observed miRNA-dependent alteration of sialyltransferases is only specific to $C$. jejuni infection requires more in-depth investigations. This will be the subject of our future research.

In the current study, we mainly focused on the interaction between ST3GAL2 and miR-615-3p, because on one hand, it showed typical binding properties by in silico analysis and on the other hand, our RT-qPCR analysis showed strongly negative correlation between both partners in colonic tissues derived from C. jejuni 81-176 infected mice. By both, the dual luciferase reporter assay and RNAi performed in CMT 93 cells, we demonstrated that miR-615-3p targets at least one predicted binding site within the $3^{\prime}$ UTR of the sialyltransferase ST3GAL2 specifically. Overall, the results suggest that miR-615-3p might play a regulatory role during $C$. jejuni infection of mice by targeting ST3GAL2. Nevertheless, the basic mechanism and regulatory pathway of miR-6153p-ST3GAL2 interaction involved in sialylation upon murine $C$. jejuni infection still needs to be clarified. We also found that the transcription of ST3GAL1 and miR$125 a-5 p$ was negatively correlated. These observations could indicate a change in the mucin structure, as one sialyltransferase is down-regulated and the other up-regulated. However, whether miR-125a-5p specifically targets ST3GAL1 and how potentially altered glycosylation patterns impact the mucin structure are the questions remain to be investigated in our future studies.

In addition, we further determined the impact of $C$. jejuni 81-176 on ST3GAL2 expression at the protein level 
by performing immunofluorescent staining and Western blotting. Consistent with our results showing that infection caused down-regulation of ST3GAL2 mRNA, we observed reduced staining for ST3GAL2 proteins in murine colonic tissue samples at day 6 p.i. We also quantified changes of ST3GAL2 protein level via Western blotting and found a trend towards slightly decreased levels in the mouse colon 6 days after C. jejuni 81-176 infection. Nevertheless, the majority of our results reveal that the expression of the sialyltransferase ST3GAL2 is regulated during infection with $C$. jejuni $81-176$ by miR-615-3p. Due to the fixation, we could not follow the mucin change by staining, as the mucus was dehydrated by paraformaldehyde fixation and thus differences e.g. in thickness could not be traced [42].

\section{Conclusions}

In conclusion, using in vivo and in vitro approaches, this study demonstrated that $C$. jejuni infection induced dysregulation of the mucin MUC2 and its mediator TFF3 mRNA as well as mucin-associated miRNAs (miR-125a-5p and miR-615-3p) in an apparently strainindependent manner. Moreover, we observed aberrant transcription of sialyltransferases ST3GAL1 and ST3GAL2 that are involved in mucin type $O$-glycan biosynthesis. Our results suggest that these mucin-associated factors interact in a coordinated manner concerted by a miRNA-dependent regulatory network during $C$. jejuni infection. Herein, ST3GAL2 has been identified as a target of miR-615-3p in vitro and for the first time we show a regulatory relationship of miR-615-3p and ST3GAL2 involved in the host cellular response to $C$. jejuni 81-176 infection in mice. Whether the mechanisms underlying these alterations during $C$. jejuni infection involve processes that are dependent or coordinated with the regulation of other predicted miRNAs remain to be addressed. For further explorations, it could be intriguing to know the possible impact of miRNA-regulated sialyltransferases on mucin structure during $C$. jejuni infection. Moreover, we will shed light on the functional effects of miR-615-3p-ST3GAL2-interaction in response to other bacterial infections in future.

\section{Methods}

\section{Bacterial strains, cell lines and culture conditions}

Campylobacter jejuni NCTC 11168 were grown as described earlier [43]. Briefly, bacteria were routinely grown on Mueller-Hinton agar (Oxoid, Munich, Germany) supplemented with $5 \%$ defibrinated sheep blood or in Brucella broth (BD, Heidelberg, Germany) at $37{ }^{\circ} \mathrm{C}$ under microaerobic conditions $\left(10 \% \mathrm{CO}_{2}, 6 \% \mathrm{O}_{2}\right.$ and $85 \% \mathrm{~N}_{2}$ ) generated by Anoxomat (Omni Life Science, Bremen, Germany). C. jejuni NCTC 11168 were grown to mid-exponential phase in BB and centrifuged $(14,000 \times g$, $5 \mathrm{~min}$ ), and re-suspended in the cell culture medium prior to infection assay in vitro. For in vivo infection, a stock solution of $C$. jejuni 81-176 strain (stored in $-80{ }^{\circ} \mathrm{C}$ ) was thawed and aliquots streaked onto Karmali agar (Oxid, Wesel, Germany) and incubated at $37{ }^{\circ} \mathrm{C}$ under microaerobic conditions for $48 \mathrm{~h}$, as previously described [44]. Bacteria were harvested with a final inoculum of $10^{9}$ bacterial cells in sterile phosphate buffered saline (PBS) (Gibco, Life Technologies, UK) immediately prior to peroral infection of mice.

The subclone HT-29/B6 [45] of the human colorectal adenocarcinoma cell line HT-29 (DSMZ_ACC299) was maintained as previously described [43]. Briefly, HT-29/ B6 cells were routinely cultured in RPMI 1640 medium (Lonza, Basel, Switzerland) supplemented with 10\% (v/v) FCS superior (Biochrom, Berlin, Germany) in $75 \mathrm{~cm}^{2}$ tissue culture flasks (Sarstedt, Nümbrecht, Germany) at $37{ }^{\circ} \mathrm{C}$ and $5 \% \mathrm{CO}_{2}$ under a humidified atmosphere until a confluence of approx. $80 \%$ was reached. For infection assays, $5 \times 10^{5}$ HT-29/B6 cells were seeded into each well of a 6 well plate (Sarstedt) and incubated for 7 days with changing the media regularly. Murine rectum carcinoma cell line CMT 93 (ECACC 89111413) was cultured as described by Jonckheere et al. [46]. Briefly, CMT 93 was maintained in high glucose $(4.5 \mathrm{~g} / \mathrm{L})$ Dulbecco's modified Eagle medium (DMEM, Lonza, Köln, Germany) supplemented with $10 \%(\mathrm{v} / \mathrm{v})$ fetal bovine serum (FBS, Biochrom, Cambridge, United Kingdom). Cultivation of cells was performed in $75 \mathrm{~cm}^{2}$ tissue culture flasks (Sarstedt, Nümbrecht, Germany) at $37{ }^{\circ} \mathrm{C}$ in a humidified $5 \% \mathrm{CO}_{2}$ atmosphere until a confluence of approx. $75 \%$ was reached. For nucleofection, $1 \times 10^{6}$ CMT 93 cells were taken from pre-cultured CMT 93 cells with $75 \%$ confluency, followed with transfection and $24 \mathrm{~h}$ incubation.

\section{Animal experiments and tissue sampling}

Mouse experiments were carried out in compliance with the European Guidelines for animal welfare (2010/63/ $\mathrm{EU})$. The protocol was approved by the commission for animal experiments headed by the "Landesasmt für Gesundheit und Soziales" (LaGeSo, Berlin, registration numbers G0172/16 and G0247/16). Animal welfare was monitored twice daily by assessment of clinical conditions. As previously described [30], secondary abiotic IL-10 $10^{-1-}$ mice (C57BL/6j background) from the Forschungseinrichtungen für Experimentelle Medizin (FEM, Charité-University Medicine Berlin) were included in the study. Infected animals (in total $n=12$ ) were perorally challenged with $0.3 \mathrm{ml}$ PBS containing $10^{9}$ colony forming units (CFU) of C. jejuni strain 81-176, while control animals (in total $n=11$ ) were challenged with 0.3 
$\mathrm{ml}$ PBS only at day 0 and 1 . Animals of both groups were matched by sex and age, respectively. Mice were strictly kept in a sterile environment to avoid contaminations. At 6 days post infection, mice were sacrificed by cervical dislocation and intestinal samples were taken under aseptic conditions following the method previously described [44]. Intestinal tissue samples were obtained in parallel from each mouse for further immunofluorescence assays. Tissue sample lysis, RNA extraction, cDNA synthesis as well as protein isolation are described below.

\section{In vitro infection assay}

Campylobacter jejuni infection using the cell line HT-29/B6 was performed as described previously [43]. Briefly, HT-29/B6 cells were infected with a suspension of approx. $1 \times 10^{9} \mathrm{CFU}$ of C. jejuni NCTC 11168 (MOI 500). Cell monolayers were infected for 1, 5 and $24 \mathrm{~h}$ post infection, respectively. Gentamicin (300 $\mathrm{ng} /$ $\mathrm{ml}$, Biochrom) protection was performed after $3 \mathrm{~h}$ to kill extracellular bacteria. Before harvesting, infected cells were washed with PBS 3 times and lysed directly in each well by addition of cell lysis buffer (Roboklon, Berlin, Germany) before total RNA was extracted as described below. Non-infected cells (negative control) were treated under the same conditions and harvested at same time points, respectively.

\section{RNA extraction, cDNA synthesis and real-time qPCR analysis}

Total RNA was isolated, and quality controlled as described earlier [3, 43]. Briefly, total RNA from HT-29/ B6 cells was extracted by using the Universal RNA/ miRNA Purification Kit (Roboklon, Berlin, Germany) according to the manufacturer's protocol. Total RNA from murine colonic tissue and CMT 93 cells was isolated with the miRVana Isolation Kit (Life Technologies, Germany). The cDNA was synthesised from individual cell or tissue samples as described earlier [47]. Basically, the isolated total RNA was treated with DNase I (RNaseFree) (NEB GmbH, Frankfurt a/M, Germany) to exclude residual genomic DNA. $1 \mu \mathrm{g}$ total RNA was reverse transcribed using $200 \mathrm{U}$ M-MuLV Reverse Transcriptase (Thermo Fisher Scientific), $0.2 \mu \mathrm{g}$ random hexamers (Thermo Fisher Scientific), $200 \mu \mathrm{M}$ of each dNTP and $1 \times$ supplied RT buffer. Control samples were treated in the same way but without M-MuLV Reverse Transcriptase to monitor the presence of genomic DNA. Quantification of mRNA as well as miRNA expression via RT-qPCR was performed as described previously [15]. Expression data was normalised with calculated geometric means of stably expressed reference genes determined beforehand using geNorm [48]. For normalisation of mRNA expression in HT-29/B6, ACTB and B2M were used as reference genes and for normalisation of miRNA expression the small RNAs SNORD44 along with SNORD47 were used as reference [49]. For normalisation of mRNA expression in mouse colon tissue HPRT and SDHA were used as reference and miRNA expression was normalised to the miRNAs miR-16 and miR-145-5p. HPRT, SDHA and B2M were used for normalisation of mRNA expression in CMT 93 cells and miRNA expression was normalised considering miR-16 and miR-145-5p as references. The relative gene expression was calculated by the $\Delta \Delta \mathrm{Ct}$ method [50] as described earlier [15]. For the cDNA synthesis and RT-qPCR analysis (miR-Q) of the miR-320-family, special RT-6 primer and reverse PCR primer (Additional file 3) with corresponding base ambiguities were generated to cover the entire members of the miR-320 family shown in Fig. 1 as described earlier [51]. Oligonucleotides used in this study were all synthesised by Sigma-Aldrich (Darmstadt, Germany) and are listed in Additional file 3.

\section{Western blotting}

Protein from colonic tissue samples of $C$. jejuni 81-176 infected secondary abiotic IL-10 ${ }^{-1-}$ mice was isolated and quantified as previously described with a few modifications [47, 52]. Briefly, tissue was lysed in cold RIPA buffer supplemented with protease inhibitor cocktail (Thermo Fisher Scientific). Protein samples were quantified using a BCA assay (Thermo Fisher Scientific). Equal amounts of protein $(30 \mu \mathrm{g})$ were separated by $12 \%$ sodium dodecyl sulphate-polyacrylamide gel electrophoresis and transferred onto a polyvinylidene fluoride membrane (PVDF) (GE Healthcare, Buckinghamshire, UK) via semidry blotting. After blocking in $5 \%(\mathrm{w} / \mathrm{v})$ bovine serum albumin (BSA, Sigma-Aldrich) in TBST (Tris-HCl-buffer with $0.1 \%(\mathrm{v} / \mathrm{v})$ tween-20) for $2 \mathrm{~h}$ at room temperature, the membranes were probed with the primary antibodies (Rabbit anti-ST3GAL2, Novus Biologicals, Colorado, USA), 1:500 in 3\% BSA in TBST at $4{ }^{\circ} \mathrm{C}$ overnight. Membranes were then washed three times in TBST for $15 \mathrm{~min}$ and subsequently incubated with horseradish peroxidase (HRP)-linked anti-rabbit IgG antibody (1:5000 in 3\% BSA in TBST; Cell Signalling Technology) for $2 \mathrm{~h}$ at room temperature. Immuno-reactive proteins were developed by using the Amersham ${ }^{\mathrm{TM}}$ ECL Select ${ }^{\mathrm{TM}}$ Western Blotting Detection Reagent (GE Healthcare). After detection of ST3GAL2, membrane was stripped and again processed for GAPDH detection with Rabbit anti-GAPDH (\#5174; Cell Signalling Technology, Danvers, MA, USA, 1:2000 in 3\% BSA in TBST) and HRP-linked anti-rabbit IgG antibody (1:5000 in 3\% BSA in TBST; Cell Signalling Technology). Protein quantification was performed by densitometry using the software BIO-1D (Vilber 
Lourmat, Marne-la-Vallée, France). Experiments were repeated at least five times.

\section{Immunofluorescence}

For immunofluorescence detection, mouse colon sampling and immunostaining were performed as described earlier with few modification [25, 44, 53]. Briefly, mouse colon samples were immediately fixed in $5 \%$ formalin and embedded in paraffin before serial sections were cut. 5 $\mu \mathrm{m}$ of paraffin sections were deparaffinated in Roticlear (Carl Roth, Karlsruhe, Germany) and rehydrated through a graded series of ethanol followed by rinsing in distilled water and PBS ( $\mathrm{pH} 7.4$, Sigma-Aldrich). Non-specific binding was blocked with $1 \%(\mathrm{v} / \mathrm{v})$ BSA (Sigma-Aldrich) in PBST $(0.1 \%(\mathrm{v} / \mathrm{v})$ Tween-20 in PBS) for $1 \mathrm{~h}$ at room temperature. Thereafter, sections were incubated with a 1:50 dilution of primary antibody, Rabbit anti-ST3GAL2 (Novus Biologicals), in PBST containing 1\% (v/v) BSA overnight at $4{ }^{\circ} \mathrm{C}$. Negative controls were performed without using the primary antibody. After three washes with PBS for 5 min, the primary antibody was detected with goat anti-rabbit IgG DyLight 594 (1: 400, \#35561; Thermo Fisher Scientific) for $1 \mathrm{~h}$ at room temperature followed by two washing steps with PBS. Nuclei were counterstained with $200 \mathrm{ng} / \mathrm{ml} 4^{\prime}$, 6-diamidin-2-phenylindol (DAPI, Sigma-Aldrich) in PBS by 3 min incubation at room temperature. Subsequently, slides were washed in PBS and mounted with Prolong ${ }^{\mathrm{TM}}$ Diamond Antifade Mountant (Life Technology). Fluorescence microscopy was performed using a Leica DMI6000B inverted microscope and the Leica LAS-X software (Leica, Wetzlar, Germany). Immunofluorescence images were taken under identical microscope and camera settings. Images were taken with background-subtraction and at least four random areas per section and more than ten images per area were selected. Experiments were carried out with three biological replicates.

\section{RNAi and luciferase reporter assay}

CMT 93 cells were cultured as described above and transfected via electroporation using the Nucleofector Technology (Lonza AG, Köln, Germany) as previously described with some modifications [3]. For nucleofection, $1 \times 10^{6}$ cells were used together with 50 pmol of hsa-miR-615 miRVana miRNA mimics (\#4464066; Fisher Scientific, Schwerte, Germany) or 50 pmol non-targeting siRNA (D-001810-03-05, Dharmacon Lafayette, CO, USA) as control. Twenty-four hours after transfection, cells were washed with PBS and lysed for RNA isolation.

Dual luciferase reporter assays were performed according to the previous study [15]. For generation of reporter plasmids, the identified target site of murine ST3GAL2 was amplified using the hybridised oligonucleotides NotI-mmuST3GAL2-ts-sense and XbaI-mmuST3GAL2ts-antisense obtained from Sigma-Aldrich (Additional file 3). The target site was cloned in pTK-Gluc (NEB $\mathrm{GmbH}$ ) using the restriction enzymes NotI and XbaI (NEB GmbH). The endotoxin-free reporter plasmid (pTKGmST3GAL2) was produced for transfection using NucleoBond Xtra Midi Plus EF (Macherey-Nagel GmbH \& Co. KG, Düren, Germany). CMT 93 cells were transfected using $1.3 \mu \mathrm{g}$ of pTKGmST3GAL2 and $200 \mathrm{ng}$ normalisation plasmid (pTK-Cluc, NEB GmbH) along with 150 pmol Pre-miR miRNA Precursor hsa-miR-615 (Life Technologies) or 150 pmol non-sense miRNA Pre-miR miRNA Precursor Negative Control \#1 (Life Technologies) as negative control. Gaussia/Cypridina Luciferase activity was detected three times for at least four independent experiments by using the Biolux Assay Kits (NEB GmbH).

\section{In silico-analysis}

For predicting miRNAs that mutually target MUC2 and TFF3, miRmap analysis [23] was performed using a score of $\geq 50$ and lists of targets were intersected using Cytoscape [54] as described earlier [24, 52]. To emphasise the biological relevance, only miRNAs were further considered that were evolutionary conserved among human, mouse and rat (Additional file 1). After evaluating anticorrelated expression between predicted miRNAs and MUC2/TFF3 in HT-29/B6, miR-125a-5p and miR-615-3p were selected for further analysis. Their targets were predicted by performing another round of miRmap analysis and lists of targets were intersected by Cytoscape (Additional file 1). KEGG pathway enrichment was performed using Cytoscape [54] and ClueGO [55]. Functionally grouped gene ontologies and pathways were determined as described previously [52] using following settings: significant KEGG pathways enrichment $(P<0.05)$, correction method: Bonferroni step down, number of genes $\geq 4$, min percentage $\geq 10.0$ and Kappa Score $\geq 0.3$.

\section{Statistical analysis}

The unpaired t-test was used in this study to compare each treatment with control. All tests were conducted applying GraphPad Prism version 6.00 (GraphPad Software, La Jolla California USA, www.graphpad.com). Asterisks in figures summarise $P$ values $\left({ }^{*} P \leq 0.05\right.$; $\left.{ }^{* * *} P \leq 0.01 ; * * * * 0.001 ;{ }^{* * * * * *} P \leq 0.0001\right)$.

\section{Supplementary Information}

The online version contains supplementary material available at https://doi. org/10.1186/s13099-021-00437-1. 
Additional file 1. Raw data of in silico analysis. Sheet 1 shows the lists of miRNAs that target MUC2 and TFF3 as well as the intersection. Sheet 2 and 3 show the miRmap analysis of the mmu-miR-615-3p and mmu-miR$125 a-5 p$ targets. Sheet 4 shows the intersection of targets.

Additional file 2. Gene enrichment analysis by means of ClueGO analysis.

Additional file 3. Oligonucleotides used in this study. Sheet 1 shows the oligonucleotide sequences of mRNAs and sheet 2 shows the oligonucleotide sequences of the selected region of ST3GAL2. Sheet 3 shows the oligonucleotide sequences of miRNAs.

Additional file 4. Immunofluorescence detection of ST3GAL2 in colonic tissue of secondary abiotic IL-10 $0^{-1-}$ mice upon C. jejuni 81-176 infection. (a, b) ST3GAL2 immunofluorescence in colonic tissue of non-infected mice out of two individual experiments (left panels: scale bar $100 \mu \mathrm{m}$, right panels: scale bar $25 \mu \mathrm{m})$. (c, d) Immunostaining of ST3GAL2 performed twice individually in colonic tissue of C. jejuni 81-176 infected mice (left panels: scale bar $100 \mu \mathrm{m}$, right panels: scale bar $25 \mu \mathrm{m}$ ). (e) Negative controls in serial sections on the same tissue area shown in Fig. 4A (left), supplementary Fig. a (middle) and Fig. b (right) (scale bar $25 \mu \mathrm{m}$ ). (f) Negative controls in serial sections for the same tissue area shown in Fig. 4B and supplementary Fig. c \& d, respectively, (scale bar $25 \mu \mathrm{m})$. ST3GAL2 was immunofluorescently labelled in red and nuclei was stained with DAPI in blue.

\section{Acknowledgements}

We would like to express our sincere thanks to Ms. Kutz-Lohroff and Ms. Nieves Hernandez for their professional support in conducting the experiments.

\section{Authors' contributions}

DX: Performed experiments, analysed data, wrote paper. LH: Performed experiments, analysed data. TA, RE, SB: Provided advice in study design, co-edited paper. MMH, GG, SS: Designed study, performed experiments, analysed data, co-wrote paper. All authors read and approved the final manuscript.

\section{Funding}

Open Access funding enabled and organized by Projekt DEAL. This work was supported by the Research Committee of the Freie Universität Berlin. The first author thanks financial support from China Scholarship Council (CSC). This work was also supported by grants from the German Federal Ministries of Education and Research (BMBF) in frame of the zoonoses research consortium PAC-Campylobacter (IP7/ 01KI1725D) and from the Federal Ministry for Economic Affairs and Energy following a resolution of the German National Parliament, Deutscher Bundestag (ZIM, ZF4117908 AJ8) to SB and MMH. The funders had no role in study design, data collection and analysis, decision to publish or preparation of the manuscript.

\section{Availability of data and materials}

All data generated or analysed during this study are included in this published article.

\section{Declarations}

Ethics approval and consent to participate

Mouse experiments were carried out in compliance with the European Guidelines for animal welfare (2010/63/EU). The protocol was approved by the commission for animal experiments headed by the "Landesasmt für Gesundheit und Soziales" (LaGeSo, Berlin, registration numbers G0172/16 and G0247/16). Animal welfare was monitored twice daily by assessment of clinical conditions.

\section{Consent for publication}

Not applicable.

\section{Competing interests}

The authors declare that they have no competing interests.

\section{Author details}

${ }^{1}$ Institute of Veterinary Biochemistry, Freie Universität Berlin, Berlin, Germany. ${ }^{2}$ Institute of Food Safety and Food Hygiene, Freie Universität Berlin,
Berlin, Germany. ${ }^{3}$ Charité - Universitätsmedizin Berlin, corporate member of Freie Universität Berlin, Humboldt-Universität zu Berlin, and Berlin Institute of Health, Institute of Microbiology, Infectious Diseases and Immunology, Berlin, Germany.

Received: 5 March 2021 Accepted: 10 June 2021

Published online: 28 June 2021

\section{References}

1. Hansson GC. Role of mucus layers in gut infection and inflammation. Curr Opin Microbiol. 2012;15(1):57-62.

2. Johansson ME, Larsson JM, Hansson GC. The two mucus layers of colon are organized by the MUC2 mucin, whereas the outer layer is a legislator of host-microbial interactions. Proc Natl Acad Sci U S A. 2011;108(Suppl 1):4659-65.

3. Hanisch C, Sharbati J, Kutz-Lohroff B, Huber O, Einspanier R, Sharbati S. TFF3-dependent resistance of human colorectal adenocarcinoma cells HT-29/B6 to apoptosis is mediated by miR-491-5p regulation of IncRNA PRINS. Cell Death Discov. 2017;3:16106.

4. Kim YS, Ho SB. Intestinal goblet cells and mucins in health and disease: recent insights and progress. Curr Gastroenterol Rep. 2010;12(5):319-30.

5. Johansson ME, Phillipson M, Petersson J, Velcich A, Holm L, Hansson GC. The inner of the two Muc2 mucin-dependent mucus layers in colon is devoid of bacteria. Proc Natl Acad Sci U S A. 2008;105(39):15064-9.

6. Mashimo H, Wu DC, Podolsky DK, Fishman MC. Impaired defense of intestinal mucosa in mice lacking intestinal trefoil factor. Science. 1996;274(5285):262-5.

7. Stone EL, Lee SH, Ismail MN, Fukuda M. Characterization of mice with targeted deletion of the gene encoding core 2 beta1,6- $\mathrm{N}$-acetylglucosaminyltransferase-2. Methods Enzymol 2010, 479:155-72.

8. Poole J, Day CJ, von Itzstein M, Paton JC, Jennings MP. Glycointeractions in bacterial pathogenesis. Nat Rev Microbiol. 2018;16(7):440-52.

9. Jensen $\mathrm{PH}$, Kolarich D, Packer NH. Mucin-type O-glycosylation-putting the pieces together. FEBS J. 2010;277(1):81-94.

10. Sicard JF, Le Bihan G, Vogeleer P, Jacques M, Harel J. Interactions of Intestinal Bacteria with Components of the Intestinal Mucus. Front Cell Infect Microbiol. 2017;7:387.

11. Naughton JA, Marino K, Dolan B, Reid C, Gough R, Gallagher ME, Kilcoyne M, Gerlach JQ, Joshi L, Rudd P, et al. Divergent mechanisms of interaction of Helicobacter pylori and Campylobacter jejuni with mucus and mucins. Infect Immun. 2013;81(8):2838-50.

12. Silva J, Leite D, Fernandes M, Mena C, Gibbs PA, Teixeira P. Campylobacter spp. as a Foodborne Pathogen: A Review. Front Microbiol. 2011;2:200.

13. Gebert LFR, MacRae IJ. Regulation of microRNA function in animals. Nat Rev Mol Cell Biol. 2019;20(1):21-37.

14. Eulalio A, Schulte L, Vogel J. The mammalian microRNA response to bacterial infections. RNA Biol. 2012;9(6):742-50.

15. Sharbati J, Lewin A, Kutz-Lohroff B, Kamal E, Einspanier R, Sharbati S Integrated microRNA-mRNA-analysis of human monocyte derived macrophages upon Mycobacterium avium subsp. hominissuis infection. PLoS One. 2011;6(5):e20258.

16. Hoeke L, Sharbati J, Pawar K, Keller A, Einspanier R, Sharbati S. Intestinal Salmonella typhimurium infection leads to miR-29a induced caveolin 2 regulation. PLoS One. 2013;8(6):e67300.

17. Sun YM, Lin KY, Chen YQ. Diverse functions of miR-125 family in different cell contexts. J Hematol Oncol. 2013;6:6.

18. Zhou X, Chen H, Zhu L, Hao B, Zhang W, Hua J, Gu H, Jin W, Zhang G: Helicobacter pylori infection related long noncoding RNA (InCRNA) AF147447 inhibits gastric cancer proliferation and invasion by targeting MUC2 and up-regulating miR-34c. Oncotarget. 2016, 7(50):82770-82.

19. Singh $N$, Shirdel EA, Waldron $L$, Zhang RH, Jurisica I, Comelli EM. The murine caecal microRNA signature depends on the presence of the endogenous microbiota. Int J Biol Sci. 2012;8(2):171-86.

20. Kasper BT, Koppolu S, Mahal LK. Insights into miRNA regulation of the human glycome. Biochem Biophys Res Commun. 2014;445(4):774-9.

21. Kurcon T, Liu Z, Paradkar AV, Vaiana CA, Koppolu S, Agrawal P, Mahal LK. miRNA proxy approach reveals hidden functions of glycosylation. Proc Natl Acad Sci USA. 2015;112(23):7327-32. 
22. Bergstrom KS, Guttman JA, Rumi M, Ma C, Bouzari S, Khan MA, Gibson DL, Vogl AW, Vallance BA. Modulation of intestinal goblet cell function during infection by an attaching and effacing bacterial pathogen. Infect Immun. 2008;76(2):796-811.

23. Vejnar CE, Zdobnov EM. MiRmap: comprehensive prediction of microRNA target repression strength. Nucleic Acids Res. 2012;40(22):11673-83.

24. Pawar K, Sharbati J, Einspanier R, Sharbati S. Mycobacterium bovis BCG interferes with miR-3619-5p control of cathepsin S in the process of autophagy. Front Cell Infect Microbiol. 2016;6:27.

25. Bereswill S, Fischer A, Plickert R, Haag LM, Otto B, Kuhl AA, Dasti I, Zautner $A E$, Munoz M, Loddenkemper C, et al. Novel murine infection models provide deep insights into the "menage a trois" of Campylobacter jejuni, microbiota and host innate immunity. PLoS ONE. 2011;6(6):e20953.

26. Kanehisa M, Goto S. KEGG: kyoto encyclopedia of genes and genomes. Nucleic Acids Res. 2000;28(1):27-30.

27. Dennis G Jr, Sherman BT, Hosack DA, Yang J, Gao W, Lane HC, Lempicki RA. DAVID: database for annotation, visualization, and integrated discovery. Genome Biol. 2003;4(5):P3.

28. Kruger J, Rehmsmeier M. RNAhybrid: microRNA target prediction easy, fast and flexible. Nucleic Acids Res. 2006, 34(Web Server issue):W451-454.

29. Erben U, Loddenkemper C, Doerfel K, Spieckermann S, Haller D, Heimesaat MM, Zeitz M, Siegmund B, Kuhl AA. A guide to histomorphological evaluation of intestinal inflammation in mouse models. Int J Clin Exp Pathol. 2014;7(8):4557-76.

30. Haag LM, Fischer A, Otto B, Plickert R, Kuhl AA, Gobel UB, Bereswill S, Heimesaat MM. Campylobacter jejuni induces acute enterocolitis in gnotobiotic IL-10-/- mice via Toll-like-receptor-2 and - 4 signaling. PLoS One. 2012;7(7):e40761.

31. Linden SK, Sutton P, Karlsson NG, Korolik V, McGuckin MA. Mucins in the mucosal barrier to infection. Mucosal Immunol. 2008;1(3):183-97.

32. Alemka A, Corcionivoschi N, Bourke B. Defense and adaptation: the complex inter-relationship between Campylobacter jejuni and mucus. Front Cell Infect Microbiol. 2012;2:15.

33. Xue $Y$, Zhang $\mathrm{H}$, Wang $\mathrm{H}, \mathrm{Hu}$ J, Du M, Zhu MJ. Host inflammatory response inhibits Escherichia coli O157:H7 adhesion to gut epithelium through augmentation of mucin expression. Infect Immun. 2014;82(5):1921-30.

34. Bereswill S, Mousavi S, Weschka D, Buczkowski A, Schmidt S, Heimesaat MM. Peroral clove essential oil treatment ameliorates acute Campylobacteriosis-results from a preclinical murine intervention study. Microorganisms. 2021, 9(4):735

35. Schmidt AM, Escher U, Mousavi S, Boehm M, Backert S, Bereswill S, Heimesaat MM. Protease activity of Campylobacter jejuni HtrA modulates distinct intestinal and systemic immune responses in infected secondary abiotic IL-10 deficient mice. Front Cell Infect Microbiol. 2019;9:79.

36. Krishn SR, Batra SK, Kaur S. Advances in miRNA-mediated mucin regulation. Curr Pharmacol Rep. 2015;1(6):355-64.

37. Eyking A, Reis H, Frank M, Gerken G, Schmid KW, Cario E. MiR-205 and MiR-373 are associated with aggressive human mucinous colorectal cancer. PLOS ONE. 2016;11(6):e0156871.

38. Kudelka MR, Stowell SR, Cummings RD, Neish AS. Intestinal epithelial glycosylation in homeostasis and gut microbiota interactions in IBD. Nat Rev Gastroenterol Hepatol. 2020;17(10):597-617.

39. McGuckin MA, Linden SK, Sutton P, Florin TH. Mucin dynamics and enteric pathogens. Nat Rev Microbiol. 2011;9(4):265-78.

40. Angata K, Fukuda M: ST3 Beta-Galactoside Alpha-2,3-Sialyltransferase 1 (ST3GAL 1). In: Taniguchi N, Honke K, Fukuda M, Narimatsu H, Yamaguchi Y, Angata T Eds. Handbook of Glycosyltransferases and Related Genes. Tokyo: Springer Japan; 2014, 637-644.

41. Tsuji S, Takashima S: ST3 Beta-Galactoside Alpha-2,3-Sialyltransferase 2 (ST3GAL2). In: Taniguchi N, Honke K, Fukuda M, Narimatsu H, Yamaguchi
Y, Angata T Eds. Handbook of Glycosyltransferases and Related Genes. Tokyo: Springer Japan; 2014, 645-656.

42. Hansson GC, Johansson ME. The inner of the two Muc2 mucindependent mucus layers in colon is devoid of bacteria. Gut Microbes. 2010;1(1):51-4

43. Xi, Alter T, Einspanier R, Sharbati S, Golz G. Campylobacter jejuni genes Cj1492C and Cj1507c are involved in host cell adhesion and invasion. Gut Pathog. 2020;12:8.

44. Mousavi S, Escher U, Thunhorst E, Kittler S, Kehrenberg C, Bereswill S, Heimesaat MM. Vitamin C alleviates acute enterocolitis in Campylobacter jejuni infected mice. Sci Rep. 2020;10(1):2921.

45. Kreusel KM, Fromm M, Schulzke JD, Hegel U. Cl- secretion in epithelial monolayers of mucus-forming human colon cells (HT-29/B6). Am J Physiol. 1991;261(4 Pt 1):C574-82.

46. Jonckheere N, Van Der Sluis M, Velghe A, Buisine MP, Sutmuller M, Ducourouble MP, Pigny P, Buller HA, Aubert JP, Einerhand AW, et al. Transcriptional activation of the murine Muc5ac mucin gene in epithelial cancer cells by TGF-beta/Smad4 signalling pathway is potentiated by Sp1. Biochem J. 2004;377(Pt 3):797-808.

47. Scholven J, Taras D, Sharbati S, Schon J, Gabler C, Huber O, Meyer zum Buschenfelde D, Blin N, Einspanier R. Intestinal expression of TFF and related genes during postnatal development in a piglet probiotic trial. Cell Physiol Biochem. 2009;23(1-3):143-56.

48. Vandesompele J, De Preter K, Pattyn F, Poppe B, Van Roy N, De Paepe A, Speleman F. Accurate normalization of real-time quantitative RT-PCR data by geometric averaging of multiple internal control genes. Genome Biol. 2002;3(7):RESEARCH0034.

49. Sharbati S, Sharbati J, Hoeke L, Bohmer M, Einspanier R. Quantification and accurate normalisation of small RNAs through new custom RT-qPCR arrays demonstrates Salmonella-induced microRNAs in human monocytes. BMC Genom. 2012;13:23.

50. Livak KJ, Schmittgen TD. Analysis of relative gene expression data using real-time quantitative PCR and the 2(-Delta Delta C(T)) Method. Methods. 2001;25(4):402-8.

51. Sharbati-Tehrani S, Kutz-Lohroff B, Bergbauer R, Scholven J, Einspanier R. miR-Q: a novel quantitative RT-PCR approach for the expression profiling of small RNA molecules such as miRNAs in a complex sample. BMC Mol Biol. 2008;9:34.

52. Pawar K, Hanisch C, Palma Vera SE, Einspanier R, Sharbati S. Down regulated IncRNA MEG3 eliminates mycobacteria in macrophages via autophagy. Sci Rep. 2016;6:19416.

53. Sharbati J, Bohmer M, Bohmer N, Keller A, Backes C, Franke A, Steinberg P, Zeljenkova D, Einspanier R. Transcriptomic analysis of intestinal tissues from Two 90-Day feeding studies in rats using genetically modified MON810 maize varieties. Front Genet. 2017;8:222.

54. Cline MS, Smoot M, Cerami E, Kuchinsky A, Landys N, Workman C, Christmas R, Avila-Campilo I, Creech M, Gross B, et al. Integration of biological networks and gene expression data using Cytoscape. Nat Protoc. 2007;2(10):2366-82.

55. Bindea G, Mlecnik B, Hackl H, Charoentong P, Tosolini M, Kirilovsky A, Fridman WH, Pages F, Trajanoski Z, Galon J. ClueGO: a Cytoscape plug-in to decipher functionally grouped gene ontology and pathway annotation networks. Bioinformatics. 2009;25(8):1091-3.

\section{Publisher's Note}

Springer Nature remains neutral with regard to jurisdictional claims in published maps and institutional affiliations. 